www.mdpi.com/journal/applsci

\title{
Communication
}

\section{Microwave-Assisted Synthesis and Antifungal Activity of Some Novel Thioethers Containing 1,2,4-Triazole Moiety}

\section{Li-Jing Min ${ }^{1, \dagger}$, Yan-Xia Shi ${ }^{2, \dagger}$, Hong-Ke Wu ${ }^{3}$, Zhao-Hui Sun ${ }^{3}$, Xing-Hai Liu ${ }^{3, *}$, Bao-Ju Li ${ }^{2, *}$ and Yong-Gang Zhang ${ }^{4, *}$}

1 College of Life Science, Huzhou University, Huzhou 313000, China; E-Mail: minlijing@hztc.zj.cn

2 Institute of Vegetables and Flowers, Chinese Academy of Agricultural Sciences, Beijing 100014, China; E-Mail: shiyanxia@caas.cn

3 College of Chemical Engineering, Zhejiang University of Technology, Hangzhou 310014, China; E-Mails: wuhk910@gmail.com (H.-K.W.); sunzhaohuizjut@163.com (Z.-H.S.)

4 Ecology Institute of Shandong Academy of Science, Jinan 250014, China

$\dagger$ These authors contributed equally to this work.

* Authors to whom correspondence should be addressed; E-Mails: xhliu@zjut.edu.cn (X.-H.L.); libaoju@caas.cn (B.-J.L.); zhangyg@sdas.org (Y.-G.Z.); Tel./Fax: +86-571-8832-0832 (X.-H.L.).

Academic Editor: Rajender S. Varma

Received: 8 October 2015 / Accepted: 11 November 2015 / Published: 16 November 2015

\begin{abstract}
A series of novel thioether derivatives containing 1,2,4-triazole moiety were designed and synthesized from 4-chlorophenol and ethyl 2-chloroacetate as starting materials by multi-step reactions under microwave irradiation, and their structures were characterized by ${ }^{1} \mathrm{H}-\mathrm{NMR}$, MS and elemental analysis. The antifungal activity of title compounds was determined. The results indicated that some of title compounds exhibited moderate antifungal activity.
\end{abstract}

Keywords: thioether; 1,2,4-triazole; microwave assistant synthesis; antifungal activity

\section{Introduction}

In recent years, heterocycles had been attracted in medicinal or pesticidal importance due to its wide and spectrum activity [1-6]. The 1,2,4-triazole compounds represent an important nitro-containing heterocycles with extensively bioactivities [7-10], such as anti-inflammatory activity [11], anticancer 
activity [12], anti-oxidation activity [13,14], herbicidal activity [15], antifungal activity [16,17], antiurease activity [18], antidepressant activity [19] and so on. Moreover, 1,2,4-triazole substructure was found in many commercial pesticide or drugs, especially in antifungal medicines (Figure 1). It is an important research field for developing novel 1,2,4-triazole derivatives. Meanwhile, the phenoxy nucleus is key fragment in many herbicides [20]. For example, it is reported that 2,4-dichlorophenoxyacetylhydrazines exhibited excellent antifungal or herbicidal activity.<smiles>CC(C)(C)C(O)/C(=C\c1ccc(Cl)cc1Cl)n1cncn1</smiles><smiles>CCCCOC(=O)COc1ccc(Cl)cc1Cl</smiles><smiles>O=C(O)COc1ccc(Cl)cc1Cl</smiles><smiles>N#Cc1ccc(C/C(=N\NC(=O)Cc2ccc(OC(F)(F)F)cc2)c2cccc(C(F)(F)F)c2)cc1</smiles>

metaflumizon

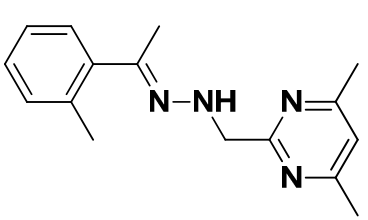

fermzone<smiles>CC1(C)CNC(=NN=C(/C=C\c2ccc(C(F)(F)F)cc2)/C=C/c2ccc(C(F)(F)F)cc2)NC1</smiles>

hydramethylnon

Figure 1. Some representative commercial drugs or pesticides.

On the other hand, 1,2,4-triazole-thiones are useful intermediates in the field of drug design and discovery. According the rule of bioisostere, when the oxygen atom was replaced by sulfur atom, the biological activity will be improved. So far, many bioactive 1,2,4-triazole-thioether or 1,2,4-triazole-thione derivatives have been reported. For example, Polucci and co-authors synthesized a series of alkylsulfanyl-1,2,4-triazoles, which exhibited excellent anticancer activity [21]. Another interesting reference reported that a series of 1,2,4-triazole-thioether derivatives containing benzothiazinone and benzooxazinone moiety displayed excellent activity as non-glucoside SGLT2 inhibitors [22]. Also, we had synthesized some interesting 1,2,4-triazole-thioether derivatives containing 1,2,3-thiadiazole [23] or pyridine [24] moiety, and these compounds possessed good antifungal activity. 
Kochikyan and co-workers [25] reported the synthesis method about 1,2,4-triazole-3-thiols and their $S$-substituted derivatives.

Microwave-assisted technique is a green method in current organic synthesis [26-28]. It is attractive offering reduced pollution, low cost and high yields. The method can often shorten the reaction time. In recent years, many references had been reported this method. For example, MW-assisted synthesis heterocycles have been conducted successfully earlier $[29,30]$.

In view of all these facts and as continuation of our research on bioactive compounds [31-33], herein a series of novel thioether derivatives containing 1,2,4-triazole moiety were synthesized under microwave irradiation. The antifungal activity of title compounds was tested.

\section{Results and Discussion}

\subsection{Synthesis}

The ethyl 2-(4-chlorophenoxy)acetate was obtained from 4-chlorophenol and ethyl 2-chloroacetate catalyzed by KI at reflux condition. The KI was used as catalyst in order to increase the yield of ethyl 2-(4-chlorophenoxy)acetate. The ethyl 2-(4-chlorophenoxy)acetate reacted with $85 \%$ hydrazine hydrate to give 2-(4-chlorophenoxy)acetohydrazide at refluxing condition. Then the 2-(4-chlorophenoxy)acetohydrazide reacted with isothiocyanatobenzene. Then 2-(2-(4-chlorophenoxy)acetyl) $-N$-phenylhydrazinecarbothioamide was collected easily by filtered. The key intermediate 5-((4-chlorophenoxy)methyl)-4-phenyl-4H-1,2,4-triazole-3-thiol was cyclized under alkaline condition, such as $\mathrm{NaOH}$, then the concentrate $\mathrm{HCl}$ was added to this solution. The key intermediatethiol is reacted with substituted benzyl chloride or alkylchloride to afford title compounds. The microwave-assisted synthesis and conventional method was also employed in this experiment. $\mathrm{NaOH} / \mathrm{DMF} / \mathrm{H}_{2} \mathrm{O}$ system was applied under microwave irradiation. The best reaction condition is at $90{ }^{\circ} \mathrm{C}$ for $15 \mathrm{~min}$ under microwave irradiation synthesis. Taking the compound $\mathbf{5 b}$ as example, we can see that the yield is higher than that of conventional method, also the reaction time is shorter from Table 1.

Table 1. Comparison of yields of $\mathbf{5 b}$ through methods with or without microwave irradiation.

\begin{tabular}{ccccc}
\hline No. & Method & Time & Temperature ${ }^{\circ} \mathbf{C}$ & Yield/\% \\
\hline $\mathbf{5 b}$ & No-MW & $24 \mathrm{~h}$ & r.t. & 78 \\
$\mathbf{5 b}$ & No-MW & $10 \mathrm{~min}$ & 90 & 42 \\
$\mathbf{5 b}$ & MW & $10 \mathrm{~min}$ & 90 & 79 \\
$\mathbf{5 b}$ & MW & $15 \mathrm{~min}$ & 90 & 81 \\
$\mathbf{5 b}$ & MW & $20 \mathrm{~min}$ & 90 & 81 \\
\hline
\end{tabular}

\subsection{Antifungal Activities}

The in vivo fungicidal results of title compounds against Pythium ultimum Trow, Phytophthora infestans (Mont.) de Bary, Corynespora cassiicola, Botrytis cinerea and Rhizoctonia solani were listed in Table 2, Zhongshengmycin, Dimethomorph, Procymidone, Chlorothalonil and Validamycin were used as controls. As shown in Table 2, some of the title compoundsshowed good control efficacy against Pythium ultimum Trow at a concentration of $100 \mu \mathrm{g} / \mathrm{mL}$, such as compound $\mathbf{5 a}(66.7 \%), \mathbf{5 b}(55.6 \%), \mathbf{5 h}$ $(55.6 \%)$ and $5 \mathbf{i}(55.6 \%)$, which is better than that of control. While some of them exhibited low activity 
against Pythium ultimum Trow. For example, compound 5c (-88.9\%), 5d (-111.1\%), 5e $(-55.6 \%), 5 j(-33.3 \%), 5 k(-122.2 \%)$ held no inhibitory against Pythium ultimum Trow. On the opposite, these compounds can increase the fungal growth. For this fungal Pythium ultimum Trow, the control Zhongshengmycin also cannot inhibit it. For the fungal Corynespora cassiicola, the compound 5c (46.2\%), 5d (46.2\%), 5e (61.3\%) and 5f (50.9\%) displayed excellent inhibition respectively. They exhibited the same inhibitory as that of the controlchlorothalonil (45.9\%).All the title compounds exhibited no inhibition effect against Rhizoctonia solani and Phytophthora infestans (Mont.) de Bary. Only compound 5a (3.9\%), 5c (6.7\%) and 5k (10.6\%) displayed weak inhibitory against Rhizoctonia solani. The control procymidone also exhibited no activity against Rhizoctonia solani. Surprisingly, most of these compounds showed stimulate growth activity against Botrytis cinerea, except compound 5c (23.8\%), 5d (19.9\%) and 5e (19.9\%).

Table 2. The antifungal activity of title compounds in vivo at $100 \mathrm{ppm}(\%)$.

\begin{tabular}{cccccc}
\hline No. & $\begin{array}{c}\text { Pythium } \\
\text { ultimum }\end{array}$ & $\begin{array}{c}\text { Phytophthora } \\
\text { infestans }\end{array}$ & $\begin{array}{c}\text { Corynespora } \\
\text { cassiicola }\end{array}$ & $\begin{array}{c}\text { Botrytis } \\
\text { cinerea }\end{array}$ & $\begin{array}{c}\text { Rhizoctonia } \\
\text { solani }\end{array}$ \\
\hline $\mathbf{5 a}$ & $66.7 \pm 2.15$ & $-0.8 \pm 0.08$ & $4.1 \pm 1.02$ & $-25.4 \pm 1.32$ & $3.9 \pm 0.05$ \\
$\mathbf{5 b}$ & $55.6 \pm 1.65$ & $-0.8 \pm 0.02$ & $-0.9 \pm 0.06$ & $-11.9 \pm 0.98$ & $0.0 \pm 0.00$ \\
$\mathbf{5 c}$ & $-88.9 \pm 2.03$ & $-0.8 \pm 0.09$ & $46.2 \pm 2.05$ & $23.8 \pm 1.05$ & $6.7 \pm 0.07$ \\
$\mathbf{5 d}$ & $-111.1 \pm 2.78$ & $-0.8 \pm 0.01$ & $46.2 \pm 2.12$ & $19.9 \pm 0.96$ & $0.0 \pm 0.00$ \\
$\mathbf{5 e}$ & $-55.6 \pm 0.89$ & $-0.8 \pm 0.04$ & $61.3 \pm 1.89$ & $19.9 \pm 1.01$ & $0.0 \pm 0.00$ \\
$\mathbf{5 f}$ & $11.1 \pm 0.78$ & $-0.8 \pm 0.03$ & $50.9 \pm 1.66$ & $-16.7 \pm 0.88$ & $0.0 \pm 0.00$ \\
$\mathbf{5 g}$ & $0.0 \pm 0.00$ & $-0.8 \pm 0.04$ & $41.4 \pm 1.15$ & $-16.7 \pm 0.79$ & $0.0 \pm 0.00$ \\
$\mathbf{5 h}$ & $55.6 \pm 1.35$ & $-0.5 \pm 0.04$ & $37.8 \pm 1.25$ & $-56.3 \pm 1.22$ & $0.0 \pm 0.00$ \\
$\mathbf{5 i}$ & $55.6 \pm 1.62$ & $-0.8 \pm 0.02$ & $24.9 \pm 2.16$ & $-15.8 \pm 1.14$ & $0.0 \pm 0.00$ \\
$\mathbf{5 j}$ & $-33.3 \pm 1.01$ & $0.3 \pm 0.04$ & $42.2 \pm 1.14$ & $-46.6 \pm 0.38$ & $0.0 \pm 0.00$ \\
$\mathbf{5 k}$ & $-122.2 \pm 3.05$ & $3.3 \pm 0.05$ & $16.2 \pm 1.18$ & $1.6 \pm 0.06$ & $10.6 \pm 0.52$ \\
Zhongshengmycin & $0.0 \pm 0.00$ & - & - & - & - \\
Dimethomorph & - & $97.8 \pm 2.07$ & - & - & - \\
Chlorothalonil & - & - & $45.9 \pm 1.77$ & - & - \\
Procymidone & - & - & - & $-7.6 \pm 0.68$ & - \\
Validamycin & - & - & - & - & $62.5 \pm 2.13$ \\
\hline
\end{tabular}

\section{Experimental Section}

\subsection{Instruments}

Melting points were recorded using an X-4 apparatus and uncorrected (Beijing Tech Instrument Co., Beijing, China). ${ }^{1} \mathrm{H}-\mathrm{NMR}$ spectra were performed on a Bruker AV-400 instrument (Bruker, Fallanden, Switzerland) using TMS as an internal standard and $\mathrm{CDCl}_{3}$ as the solvent. Elemental analyses were determined on a Vario EL elemental analyzer (Elementar, Hanau, Germany). All the reagents are of analytical grade or freshly prepared before use.

\subsection{Synthesis}

The synthetic procedure for title compound is shown in Scheme 1. 

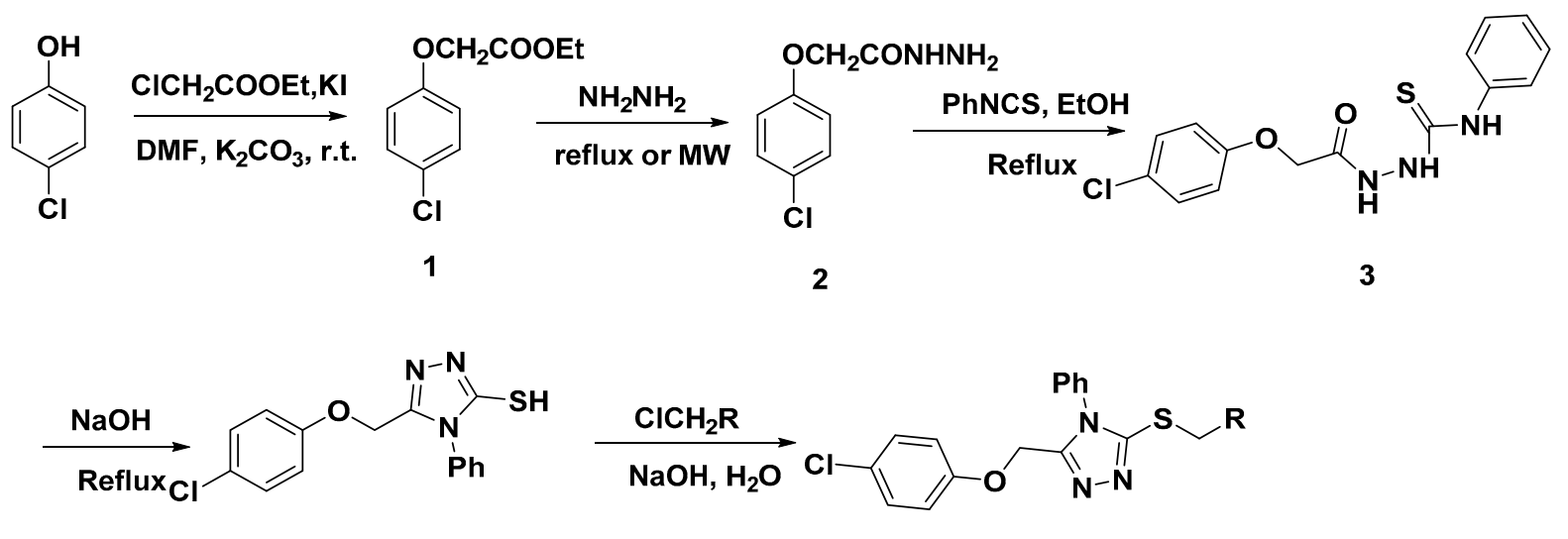

4

5

Scheme 1. The synthetic route of title compounds.

\subsubsection{Synthesis of Ethyl 2-(4-chlorophenoxy)acetate (1)}

4-Chlorophenol $(0.1 \mathrm{~mol})$, acetone $(40 \mathrm{~mL})$, ethyl 2-chloroacetate $(0.12 \mathrm{~mol}), \mathrm{K}_{2} \mathrm{CO}_{3}(16.56 \mathrm{~g}$, $0.12 \mathrm{~mol}$ ) and catalytic amount of KI were added to a $100 \mathrm{~mL}$ three-necked flask, then the mixture was stirred at reflux. The reaction was monitored by GC until the start material la disappeared completely. The mixture was poured into ice water and extracted with ethyl acetate $(3 \times 50 \mathrm{~mL})$ and then dried by anhydrous $\mathrm{Na}_{2} \mathrm{SO}_{4}$ and evaporated to dryness to get crude solid $\mathbf{1}$.

\subsubsection{Synthesis of 2-(4-Chlorophenoxy)acetohydrazide (2)}

A mixture of ethyl 2-(4-chlorophenoxy)acetate $(1.44 \mathrm{~g}, 10 \mathrm{mmol})$ and $85 \%$ hydrazine hydrate $(2 \mathrm{~mL}$, $35 \mathrm{mmol}$ ) was heated under reflux for $6 \mathrm{~h}$. The mixture was cooled to room temperature, filtered, washed with cool ethyl acetate, then dried to give white solid 2-(4-chlorophenoxy)acetohydrazide 2, yield 83\%.

\subsubsection{Synthesis of 2-(2-(4-Chlorophenoxy)acetyl)- $N$-phenylhydrazinecarbothioamide (3)}

A mixture of 2-(4-chlorophenoxy)acetohydrazide $(10 \mathrm{mmol})$ with isothiocyanatobenzene $(10 \mathrm{mmol})$ was refluxed for $5 \mathrm{~h}$ in ethanol. After cooling down to room temperature, the products were obtained and recrystallized from methanol to give 3 , yield $95 \%$.

\subsubsection{Synthesis of 5-((4-Chlorophenoxy)methyl)-4-phenyl-4H-1,2,4-triazole-3-thiol (4)}

A mixture of compound (3) $(10 \mathrm{mmol})$ in aqueous $\mathrm{NaOH}$ solution $(5 \mathrm{~mL}, 2 \mathrm{~N})$ was refluxed for $4 \mathrm{~h}$. After cooling down to room temperature, $\mathrm{HCl}$ aqueous solution $(4 \mathrm{~N})$ was added to afford a large amount of precipitate. The solid was filtered, dried and recrystallized from methanol to give intermediate 4, yield $88 \%$.

\subsubsection{General Procedure for Thioether (5)}

A CEM designed $10 \mathrm{~mL}$ pressure-rated vial was charged with DMF $(5 \mathrm{~mL}), 4(1 \mathrm{mmol}), \mathrm{RCH}_{2} \mathrm{Cl}$ $(1.1 \mathrm{mmol})$ and $\mathrm{NaOH}(1.2 \mathrm{mmol})$. The mixture was irradiated in a CEM Discover Focused Synthesizer $\left(150 \mathrm{~W}, 90{ }^{\circ} \mathrm{C}, 200 \mathrm{psi}, 15 \mathrm{~min}\right)$. The mixture was cooled to room temperature by passing compressed 
air through the microwave cavity for $2 \mathrm{~min}$. It was poured into cold ice $(40 \mathrm{~mL})$ and the formed precipitate was filtered. The crude solid was recrystallized from EtOH to give the title compounds 5a. All the other compounds are synthesized according to the procedure (Scheme 1).

3-(butylthio)-5-((4-chlorophenoxy)methyl)-4-phenyl-4H-1,2,4-triazole 5a yield 87\%, m.p. 140-141 ${ }^{\circ} \mathrm{C}$; ${ }^{1} \mathrm{H}-\mathrm{NMR}$ (DMSO-d6, $400 \mathrm{MHz}$ ), $\delta: 0.85$ (t, 3H, CH3), 1.34 (q, 2H, CH2), $1.62\left(\mathrm{~m}, 2 \mathrm{H}, \mathrm{CH}_{2}\right), 3.12$ (m, 2H, $\left.\mathrm{CH}_{2}\right), 3.12$ (t, 2H, SCH$), 5.09$ (s, 2H, $\left.\mathrm{OCH}_{2}\right), 6.90$ (d, J=8.2 Hz, 2H, Ph), 7.27 (d, J= 8.2 Hz, $2 \mathrm{H}, \mathrm{Ph}), 7.45-7.55(\mathrm{~m}, 5 \mathrm{H}, \mathrm{Ar}-\mathrm{H})$. ESI-MS: $375[\mathrm{M}+\mathrm{H}]^{+}$; Elemental analysis for $\mathrm{C}_{19} \mathrm{H}_{20} \mathrm{ClN}_{3} \mathrm{OS}$ : found C 60.98, H 5.56, N 11.43; calcd. C, 61.03; H, 5.39; N, 11.24.

3-(benzylthio)-5-((4-chlorophenoxy)methyl)-4-phenyl-4H-1,2,4-triazole 5b yield 81\%, m.p. 104-105 ${ }^{\circ} \mathrm{C}$; ${ }^{1} \mathrm{H}-\mathrm{NMR}$ (DMSO-d6, $400 \mathrm{MHz}$ ), $\delta: 4.38$ (s, 2H, SCH 2$), 5.08$ (s, 2H, OCH ), 6.87 (d, J=8.0 Hz, 2H, Ph), 7.27-7.20 (m, 8H, Ph), 7.51 (m, 4H, Ph). ESI-MS: $409[\mathrm{M}+\mathrm{H}]^{+}$; Elemental analysis for $\mathrm{C}_{22} \mathrm{H}_{18} \mathrm{ClN}_{3} \mathrm{OS}$ : found C 64.44, H 4.53, N 10.98; calcd. C, 64.78; H, 4.45; N, 10.30.

3-((4-chlorobenzyl)thio)-5-((4-chlorophenoxy)methyl)-4-phenyl-4H-1,2,4-triazole 5c yield 77\%, m.p. $108-109{ }^{\circ} \mathrm{C} ;{ }^{1} \mathrm{H}-\mathrm{NMR}$ (DMSO-d6, $400 \mathrm{MHz}$ ), $\delta$ : 4.38 (s, 2H, $\mathrm{SCH}_{2}$ ), 5.09 (s, 2H, $\mathrm{OCH}_{2}$ ), 6.88 (d, $J=8.2 \mathrm{~Hz}, 2 \mathrm{H}, \mathrm{Ph}), 7.05-7.19$ (m, 2H, Ph), 7.27-7.37 (m, 6H, Ph), 7.50-7.52 (m, 3H, Ph). ESI-MS: $443[\mathrm{M}+\mathrm{H}]^{+}$; Elemental analysis for $\mathrm{C}_{22} \mathrm{H}_{17} \mathrm{Cl}_{2} \mathrm{~N}_{3} \mathrm{OS}$ : found $\mathrm{C} 59.83, \mathrm{H}$ 3.97, N 9.45; calcd. C, 59.73; H, 3.87; N, 9.50.

3-((4-bromobenzyl)thio)-5-((4-chlorophenoxy)methyl)-4-phenyl-4H-1,2,4-triazole 5d yield 68\%, m.p. $99-100{ }^{\circ} \mathrm{C}$; ${ }^{1} \mathrm{H}-\mathrm{NMR}$ (DMSO- $d_{6}, 400 \mathrm{MHz}$ ), $\delta: 4.35$ (s, 2H, $\mathrm{SCH}_{2}$ ), 5.08 (s, 2H, $\mathrm{OCH}_{2}$ ), 6.88 (d, $J=8.2 \mathrm{~Hz}, 2 \mathrm{H}, \mathrm{Ph}), 7.27-7.33$ (m, 6H, Ph), 7.45-7.52 (m, 6H, Ph). ESI-MS: $488[\mathrm{M}+\mathrm{H}]^{+}$; Elemental analysis for $\mathrm{C}_{22} \mathrm{H}_{17} \mathrm{BrClN}_{3} \mathrm{OS}$ : found $\mathrm{C} 53.97, \mathrm{H} 3.31, \mathrm{~N} 8.99$; calcd. C, 54.28; H, 3.52; N, 8.63.

3-((2-chlorobenzyl)thio)-5-((4-chlorophenoxy)methyl)-4-phenyl-4H-1,2,4-triazole 5e yield 77\%, m.p. $108-109{ }^{\circ} \mathrm{C} ;{ }^{1} \mathrm{H}-\mathrm{NMR}$ (DMSO-d6, $400 \mathrm{MHz}$ ), $\delta$ : 4.44 (s, 2H, $\mathrm{SCH}_{2}$ ), 5.09 (s, 2H, $\mathrm{OCH}_{2}$ ), 6.88 (d, $J=8.2 \mathrm{~Hz}, 2 \mathrm{H}, \mathrm{Ph}), 7.08-7.19$ (m, 2H, Ph), 7.27-7.39 (m, 6H, Ph), 7.50-7.52 (m, 3H, Ph). ESI-MS: $443[\mathrm{M}+\mathrm{H}]^{+}$; Elemental analysis for $\mathrm{C}_{22} \mathrm{H}_{17} \mathrm{Cl}_{2} \mathrm{~N}_{3} \mathrm{OS}$ : found $\mathrm{C} 59.63, \mathrm{H} \mathrm{3.97,} \mathrm{N} \mathrm{9.45;} \mathrm{calcd.} \mathrm{C,} \mathrm{59.73;}$ H, 3.87; N, 9.50.

3-((4-chlorophenoxy)methyl)-5-((4-methoxybenzyl)thio)-4-phenyl-4H-1,2,4-triazole 5f yield 82\%, m.p. $122-123{ }^{\circ} \mathrm{C} ;{ }^{1} \mathrm{H}-\mathrm{NMR}$ (DMSO-d6, $400 \mathrm{MHz}$ ), $\delta: 3.72$ (s, 3H, $\mathrm{OCH}_{3}$ ), 4.33 (s, 2H, $\mathrm{SCH}_{2}$ ), 5.09 $\left(\mathrm{s}, 2 \mathrm{H}, \mathrm{OCH}_{2}\right), 6.82(\mathrm{~d}, J=6.8 \mathrm{~Hz}, 2 \mathrm{H}, \mathrm{Ph}), 6.88$ (d, $\left.J=6.8 \mathrm{~Hz}, 2 \mathrm{H}, \mathrm{Ph}\right), 7.21-7.33$ (m, 6H, Ph), 7.50-7.52 (m, 3H, Ph). ESI-MS: $439[\mathrm{M}+\mathrm{H}]^{+}$; Elemental analysis for $\mathrm{C}_{23} \mathrm{H}_{20} \mathrm{ClN}_{3} \mathrm{O}_{2} \mathrm{~S}$ : found $\mathrm{C}$ 63.12, H 4.75, N 9.86; calcd. C, 63.08; H, 4.60; N, 9.59 .

3-((4-chlorophenoxy)methyl)-5-((2,4-dichlorobenzyl)thio)-4-phenyl-4H-1,2,4-triazole 5g yield 81\%, m.p.120-121 ${ }^{\circ} \mathrm{C} ;{ }^{1} \mathrm{H}-\mathrm{NMR}$ (DMSO-d6, $400 \mathrm{MHz}$ ), $\delta: 4.41$ (s, 2H, $\mathrm{SCH}_{2}$ ), 5.09 (s, 2H, OCH ), 6.88 (d, $J=8.0 \mathrm{~Hz}, 2 \mathrm{H}, \mathrm{Ph}), 7.27$ (d, $J=8.0 \mathrm{~Hz}, 2 \mathrm{H}, \mathrm{Ph}), 7.28-7.33$ (m, 3H, Ph), 7.46-7.58 (m, 5H, Ph). ESI-MS: $478[\mathrm{M}+\mathrm{H}]^{+}$; Elemental analysis for $\mathrm{C}_{22} \mathrm{H}_{16} \mathrm{Cl}_{3} \mathrm{~N}_{3} \mathrm{OS}$ : found $\mathrm{C} 55.22, \mathrm{H} \mathrm{3.07}$, N 8.51; calcd. C, 55.42; H, 3.38; N, 8.81.

4-(((5-((4-chlorophenoxy)methyl)-4-phenyl-4H-1,2,4-triazol-3-yl)thio)methyl)benzonitrile 5h yield 79\%, m.p. 123-124 ${ }^{\circ} \mathrm{C} ;{ }^{1} \mathrm{H}-\mathrm{NMR}$ (DMSO- $d_{6}, 400 \mathrm{MHz}$ ), $\delta: 4.46$ (s, 2H, $\mathrm{SCH}_{2}$ ), 5.09 (s, 2H, $\mathrm{OCH}_{2}$ ), $6.88(\mathrm{~d}, J=6.8 \mathrm{~Hz}, 2 \mathrm{H}, \mathrm{Ph}), 7.27$ (d, $J=6.8 \mathrm{~Hz}, 2 \mathrm{H}, \mathrm{Ph}), 7.34-7.74(\mathrm{~m}, 9 \mathrm{H}, \mathrm{Ph})$. ESI-MS: $434[\mathrm{M}+\mathrm{H}]^{+}$; Elemental analysis for $\mathrm{C}_{23} \mathrm{H}_{17} \mathrm{ClN} 4 \mathrm{OS}$ : found $\mathrm{C} 63.88, \mathrm{H} 3.90, \mathrm{~N} 13.04$; calcd. C, 63.81; H, 3.96; N, 12.94. 
3-((3-chlorobenzyl)thio)-5-((4-chlorophenoxy)methyl)-4-phenyl-4H-1,2,4-triazole 5i yield 77\%, m.p. 108-109 ${ }^{\circ} \mathrm{C} ;{ }^{1} \mathrm{H}-\mathrm{NMR}$ (DMSO-d6, $400 \mathrm{MHz}$ ), $\delta$ : 4.37 (s, 2H, SCH 2$), 5.09$ (s, 2H, OCH $), 6.88$ (d, $J=8.7 \mathrm{~Hz}, 2 \mathrm{H}, \mathrm{Ph}), 7.27$ (d, $J=8.7 \mathrm{~Hz}, 2 \mathrm{H}, \mathrm{Ph}), 7.33-7.35$ (m, 3H, Ph), 7.51-7.62 (m, 5H, Ph). ESI-MS: $443[\mathrm{M}+\mathrm{H}]^{+}$; Elemental analysis for $\mathrm{C}_{22} \mathrm{H}_{17} \mathrm{Cl}_{2} \mathrm{~N}_{3} \mathrm{OS}$ : found $\mathrm{C} 59.54, \mathrm{H} 3.97$, N 9.45; calcd. C, 59.73; H, 3.87; N, 9.50.

3-((4-chlorophenoxy)methyl)-5-((2-fluorobenzyl)thio)-4-phenyl-4H-1,2,4-triazole $5 \mathbf{j}$ yield $83 \%$, m.p. $110-11{ }^{\circ} \mathrm{C} ;{ }^{1} \mathrm{H}-\mathrm{NMR}$ (DMSO-d6, $400 \mathrm{MHz}$ ), $\delta$ : 4.37 (s, 2H, $\mathrm{SCH}_{2}$ ), 5.09 (s, 2H, $\mathrm{OCH}_{2}$ ), 6.88 $(\mathrm{d}, J=7.8 \mathrm{~Hz}, 2 \mathrm{H}, \mathrm{Ph}), 7.10-7.52(\mathrm{~m}, 11 \mathrm{H}, \mathrm{Ph})$. ESI-MS: $427[\mathrm{M}+\mathrm{H}]^{+}$; Elemental analysis for $\mathrm{C}_{22} \mathrm{H}_{17} \mathrm{ClFN}_{3} \mathrm{OS}$ : found C 62.89, H 3.88, N 9.86; calcd. C, 62.04; H, 4.02; N, 9.87.

3-((4-(tert-butyl)benzyl)thio)-5-((4-chlorophenoxy)methyl)-4-phenyl-4H-1,2,4-triazole $5 \mathbf{k}$ yield 81\%, m.p. 62-63 ${ }^{\circ} \mathrm{C} ;{ }^{1} \mathrm{H}-\mathrm{NMR}$ (DMSO- $d_{6}, 400 \mathrm{MHz}$ ), $\delta: 1.25(\mathrm{~s}, 9 \mathrm{H}, \mathrm{Bu}), 4.33$ (s, 2H, $\mathrm{SCH}_{2}$ ), 5.09 $\left(\mathrm{s}, 2 \mathrm{H}, \mathrm{OCH}_{2}\right), 6.88(\mathrm{~d}, J=8.6 \mathrm{~Hz}, 2 \mathrm{H}, \mathrm{Ph}), 7.17(\mathrm{~d}, J=8.0 \mathrm{~Hz}, 2 \mathrm{H}, \mathrm{Ph}), 7.24-7.29$ (m, 5H, Ph), 7.48-7.52 (m, 4H, Ph). ESI-MS: $465[\mathrm{M}+\mathrm{H}]^{+}$; Elemental analysis for $\mathrm{C}_{26} \mathrm{H}_{26} \mathrm{ClN}_{3} \mathrm{OS}$ : found $\mathrm{C}$ 67.13, H 5.75, N 9.23; calcd. C, 67.30; H, 5.65; N, 9.06.

\subsection{Antifungal Activity}

Antifungal activity of compounds 5a-5k against Pythium ultimum Trow, Phytophthora infestans (Mont.) de Bary, Corynespora cassiicola, Botrytis cinerea and Rhizoctonia solani were evaluated according to reference [3]. A potted plant test method was adopted. Germination was conducted by soaking cucumber seeds in water for $2 \mathrm{~h}$ at $50{ }^{\circ} \mathrm{C}$ and then keeping the seeds moist for $24 \mathrm{~h}$ at $28{ }^{\circ} \mathrm{C}$ in an incubator. When the radicles were $0.5 \mathrm{~cm}$, the seeds were grown in plastic pots containing a $1: 1(v / v)$ mixture of vermiculite and peat. Cucumber plants used for inoculations were at the stage of two cotyledons, and tomato plants were five euphyllas. Tested compounds and commercial fungicides were sprayed with a hand sprayer on the surface of the leaves and on a fine morning, at the standard concentration of $100 \mu \mathrm{g} / \mathrm{mL}$, and each plant was sprayed compounds and commercial fungicides $200 \mu \mathrm{L}$. Dimethomorph, Fludioxonil, Chlorothalonil, Validamycin, Zhongshengmycin were used as a control. After $2 \mathrm{~h}$, inoculations of Phytophthora infestans, Corynespora cassiicola and Botrytis cinerea were carried out by spraying fungal spore suspension with $1 \times 10^{4}$ spore/mL, inoculation of Rhizoctonia solani and Pythium ultimum were carried out by spraying mycelial suspension of $2 \times 10^{4} \mathrm{CFU} / \mathrm{mL}$, which was smashed with IKA T10 basic ULTRA-TURRAX ${ }^{\circledR}$ (Guangzhou, China). Each kind of inoculum was sprayed $300 \mu \mathrm{L} /$ plant. Each treatment was replicated 4 times. After inoculation, the plants were maintained at $18-30{ }^{\circ} \mathrm{C}$ (mean temperature of $24{ }^{\circ} \mathrm{C}$ and above $80 \%$ relative humidity $(\mathrm{RH})$ ). The antifungal activity were evaluated when the nontreated plant (blank) fully developed symptoms. The area of inoculated treated leaves covered by disease symptoms was assessed and compared to that of nontreated ones to determine the average disease index. The relative control efficacy of compounds compared to the blank assay was calculated via the following equation:

$$
\text { relative control efficacy }(\%)=(C K-P T) / C K \times 100 \%
$$

where $C K$ is the average disease index during the blank assay and $P T$ is the average disease index after treatment during testing. All experiments were replicated three times. 


\section{Conclusions}

Some interesting thioether derivatives containing 1,2,4-triazole moiety were designed and synthesized. Their structures were confirmed by NMR, MS and elemental analysis. The primarily antifungal activities showed that some of them exhibited excellent antifungal activities.

\section{Acknowledgments}

This work was supported financially by Zhejiang Provincial Natural Science Foundation of China (No. LY16C140007) and National Key Technologies R \& D Program (2011BAE06B03-01).

\section{Author Contributions}

Li-Jing Min, Yan-Xia Shi, Hong-Ke Wu and Zhao-Hui Sun carried out experimental work, Li-Jing Min prepared the manuscript, Xing-Hai Liu, Bao-Ju Li andYong-Gang Zhang designed the material and supervised the project.

\section{Conflicts of Interest}

The authors declare no conflict of interest.

\section{References}

1. Yan, S.L.; Yang, M.Y.; Sun, Z.H.; Min, L.J.; Tan, C.X.; Weng, J.Q.; Wu, H.K.; Liu, X.H. Synthesis and antifungal activity of 1,2,3-thiadiazole derivatives containing 1,3,4-thiadiazole moiety. Lett. Drug Des. Discov. 2014, 11, 940-943.

2. Owen, W.J.; Sullenberger, M.T.; Loso, M.R.; Meyer, K.G.; Slanec, T.J. Synthesis and antifungal activity of 3-aryl-1,2,4-triazin-6-one derivatives. Pest Manag. Sci. 2015, 71, 83-90.

3. Zhang, L.J.; Yang, M.Y.; Sun, Z.H.; Tan, C.X.; Weng, J.Q.; Wu, H.K.; Liu, X.H. Synthesis and antifungal activity of 1,3,4-thiadiazole derivatives containing pyridine group. Lett. Drug Des. Discov. 2014, 11, 1107-1111.

4. Nugent, B.M.; Buysse, A.M.; Loso, M.R.; Babcock, J.M.; Johnson, T.C.; Oliver, M.P.; Martin, T.P.; Ober, M.S.; Breaux, N.; Robinson, A. Expanding the structure-activity relationship of sulfoxaflor: The synthesis and biological activity of $N$-heterocyclic sulfoximines. Pest Manag. Sci. 2015, 71, 928-936.

5. Liu, X.H.; Tan, C.X.; Weng, J.Q. Synthesis, dimeric crystal, and fungicidal activity of 1-(4-methylphenyl)-2-(5-((3,5-dimethyl-1H-pyrazol-1-yl)methyl)-4-phenyl-4H-1,2,4-triazol-3-ylthio) ethanone. Phosphorus Sulfur Silicon Relat. Elem. 2011, 186, 558-564.

6. Aggarwal, N.; Kumar, R.; Srivastava, C.; Dureja, P.; Khurana, J.M. Synthesis, biological activities and SAR studies of novel 1-ethyl-7-methyl-4-oxo-1,4-dihydro-[1,8]naphthyridine-3-carboxylic acid based diacyl and sulfonyl acyl hydrazines. Pest Manag. Sci. 2014, 70, 1071-1082.

7. Liu, X.H.; Tan, C.X.; Weng, J.Q. Phase transfer-catalyzed, one-potsynthesis of some novel $N$-pyrimidinyl- $N$ '-picotinylthioureaderivatives. Phosphorus Sulfur Silicon Relat. Elem. 2011, 186, $552-557$. 
8. Yang, M.Y.; Zhai, Z.W.; Sun, Z.H.; Yu, S.J.; Liu, X.H.; Weng, J.Q.; Tan, C.X.; Zhao, W.G. A facile one-pot synthesis of novel 1,2,4-triazolo[4,3-a]pyridine derivativescontaining the trifluoromethyl moiety using microwave irradiation. J. Chem. Res. 2015, 39, 521-523.

9. Liu, X.H.; Weng, J.Q.; Wang, B.L.; Li, Y.H.; Tan, C.X.; Li, Z.M. Microwave-assisted synthesis of novel fluorinated 1,2,4-triazole derivatives, and study of their biological activity. Res. Chem. Intermed. 2014, 40, 2605-2612.

10. Zhang, L.J.; Yang, M.Y.; Hu, B.Z.; Sun, Z.H.; Liu, X.H.; Weng, J.Q.; Tan, C.X.; Microwave-assisted synthesis of novel 8-chloro-[1,2,4]triazolo[4,3-a]pyridinederivatives. Turk. J. Chem. 2015, 39, 867-873.

11. Paprocka, R.; Wiese, M.; Eljaszewicz, A.; Helmin-Basa, A.; Modzelewska-Banachiewicz, B.; Gzella, A.; Michalkiewicz, J. Synthesis and anti-inflammatory activity of new 1,2,4-triazole derivatives. Bioorganic Med. Chem. Lett. 2015, 25, 2664-2667.

12. Plech, T.; Kaproń, B.; Paneth, A.; Kosikowska, U.; Malm, A.; Strzelczyk, A.; Stączek, P.; Świątek, Ł.; Rajtar, B.; Polz-Dacewicz, M. Determination of the Primary Molecular Target of 1,2,4-Triazole-Ciprofloxacin Hybrids. Molecules 2015, 20, 6254-6272.

13. Barbuceanu, S.-F.; Ilies, D.C.; Saramet, G.; Uivarosi, V.; Draghici, C.; Radulescu, V. Synthesis and Antioxidant Activity Evaluation of New Compounds from Hydrazinecarbothioamide and 1,2,4-Triazole Class Containing Diarylsulfone and 2,4-Difluorophenyl Moieties. Int. J. Mol. Sci. 2014, 15, 10908-10925.

14. Al-Omair, M.A.; Sayed, A.R.; Youssef, M.M. Synthesis of Novel Triazoles, Tetrazine, Thiadiazoles and Their Biological Activities. Molecules 2015, 20, 2591-2610.

15. Liu, X.H.; Xu, X.Y.; Tan, C.X.; Weng, J.Q.; Xin, J.H.; Chen, J. Synthesis, crystal structure, herbicidal activities and 3D-QSAR study of some novel 1,2,4-triazolo[4,3-a]pyridine derivatives. Pest Manag. Sci. 2015, 71, 292-301.

16. Yang, M.Y.; Zhao, W.; Liu, X.H.; Tan, C.X.; Weng, J.Q. Synthesis, crystal structure and antifungal activity of 4-(5-((2,4-dichlorobenzyl)thio)-4-phenyl-4H-1,2,4-triazol-3-yl)pyridine. Chin. J. Struct. Chem. 2015, 34, 203-207.

17. Liu, X.H.; Sun, Z.H.; Yang, M.Y.; Tan, C.X.; Weng, J.Q.; Zhang, Y.G.; Ma, Y. Microwave assistant one pot synthesis, crystal structure, antifungal activities and 3D-QSAR of novel 1,2,4-triazolo[4,3-a]pyridines. Chem. Biol. Drug Des. 2014, 84, 342-347.

18. Sokmen, B.B.; Gumrukcuoglu, N.; Ugras, S.; Sahin, H.; Sagkal, Y.; Ugras, H.I. Synthesis, Antibacterial, Antiurease, and Antioxidant Activities of Some New 1,2,4-Triazole Schiff Base and Amine Derivatives. App. Biochem. Biotechnol. 2015, 175, 705-714.

19. Radhika, C.; Venkatesham, A.; Sarangapani, M. Synthesis and antidepressant activity of di substituted-5-aryl-1,2,4-triazoles. Med. Chem. Res. 2012, 21, 3509-3513.

20. Liu, X.H.; Pan, L.; Ma, Y.; Weng, J.Q.; Tan, C.X.; Li, Y.H.; Shi, Y.X.; Li, B.J.; Li, Z.M.; Zhang, Y.G. Design, synthesis, biological activities, and 3D-QSAR of new $N, N^{\prime}$-diacylhydrazines containing 2-(2,4-dichlorophenoxy)propane moiety. Chem. Biol. Drug Des. 2011, 78, 689-694.

21. Polucci, P.; Magnaghi, P.; Angiolini, M.; Asa, D.; Avanzi, N.; Badari, A.; Bertrand, J.; Casale, E.; Cauteruccio, S.; Cirla, A.; et al. Alkylsulfanyl-1,2,4-Triazoles, a New Class of Allosteric Valosine Containing Protein Inhibitors. Synthesis and Structure-Activity Relationships. J. Med. Chem. 2013, $56,437-450$. 
22. Li, A.R.; Zhang, J.; Greenberg, J.; Lee, T.; Liu, J. Discovery of non-glucoside SGLT2 inhibitors. Bioorganic Med. Chem. Lett. 2011, 21, 2472-2475.

23. Sun, N.-B.; Fu, J.-Q.; Weng, J.-Q.; Jin, J.-Z.; Tan, C.-X.; Liu, X.-H. Microwave assisted synthesis, antifungal activity and DFT theoretical study of some novel 1,2,4-triazole derivatives containing the 1,2,3-thiadiazole moiety. Molecules 2013, 18, 12725-12739.

24. Sun, G.-X.; Yang, M.-Y.; Shi, Y.-X.; Sun, Z.-H.; Liu, X.-H.; Wu, H.-K.; Li, B.-J.; Zhang, Y.-G. Microwave assistant synthesis, antifungal activity and DFT theoretical study of some novel 1,2,4-triazole derivatives containing pyridine moiety. Int. J. Mol. Sci. 2014, 15, 8075-8090.

25. Kochikyan, T.V.; Samvelyan, M.A.; Arutyunyan, V.S.; Avetisyan, A.A.; Tamazyan, R.A.; Aivazyan, A.G. Synthesis of 1,2,4-Triazole-3-Thiols and Their $S$-Substituted Derivatives. Rus. J. Org. Chem. 2010, 46, 551-555.

26. Liu, X.H.; Zhai, Z.W.; Xu, X.Y.; Yang, M.Y.; Sun, Z.H.; Weng, J.Q.; Tan, C.X.; Chen, J. Facile and efficient synthesis and herbicidal activity determination of novel 1,2,4-triazolo[4,3-a]pyridin-3(2H)-one derivatives via microwave irradiation. Bioorganic Med. Chem. Lett. 2015, 25, 5524-5528.

27. Hassan, H.M.A.; Harakeh, S.; Sakkaf, K.A.; Denetiu, I. Progress in microwave-aided chemical synthesis. Aus. J. Chem. 2012, 65, 1647-1654.

28. Mu, J.-X.; Yang, M.-Y.; Sun, Z.-H.; Tan, C.-X.; Weng, J.-Q.; Wu, H.-K.; Liu, X.-H. Synthesis, Crystal Structure and DFT Studies of 8-chloro-3-((3-chlorobenzyl)thio)-[1,2,4]triazolo[4,3-a]pyridine. Crystals 2015, 5, 491-500.

29. Polshettiwar, V.; Varma, R.S. Greener and Sustainable Approaches to the Synthesis of Pharmaceutically Active Heterocycles. Curr. Opin. Drug Discov. Dev. 2007, 10, 723-737.

30. Polshettiwar, V.; Varma, R.S. Greener and Expeditious Synthesis of Bio-Active Heterocycles using Microwave Irradiation. Pure Appl. Chem. 2008, 80, 777-790.

31. Zhai, Z.W.; Yang, M.Y.; Sun, Z.H.; Liu, X.H.; Weng, J.Q.; Tan, C.X. Facile and efficient synthesis of novel 1,2,3-thiadiazole derivatives using microwave irradiation. J. Chem. Res. 2015, 39, 340-342.

32. Yang, M.Y.; Zhao, W.; Sun, Z.H.; Tan, C.X.; Weng, J.Q.; Liu, X.H. Synthesis and Biological Activity of Acylthiourea Derivatives Contain 1,2,3-Thiadiazole and 1,3,4-Thiadiazole. Lett. Drug Des. Discov. 2015, 12, 314-318.

33. Tan, C.X.; Weng, J.Q.; Liu, Z.X.; Liu, X.H.; Zhao, W.G. Synthesis, crystal structure and fungicidal activity of a novel 1,2,3-thiadiazole compound. Phosphorus Sulfur Silicon Relat. Elem. 2012, 187, 990-996.

(C) 2015 by the authors; licensee MDPI, Basel, Switzerland. This article is an open access article distributed under the terms and conditions of the Creative Commons Attribution license (http://creativecommons.org/licenses/by/4.0/). 